\title{
Decomposing complete equipartite graphs into short odd cycles
}

\author{
Benjamin R. Smith \\ Centre for Discrete Mathematics and Computing \\ Department of Mathematics \\ The University of Queensland \\ Qld 4072, Australia \\ bsmith@maths.uq.edu.au \\ Nicholas J. Cavenagh \\ The Department of Mathematics \\ The University of Waikato \\ Private Bag 3105 \\ Hamilton, New Zealand \\ School of Mathematical Sciences \\ Monash University \\ Vic 3800, Australia \\ nickc@math.waikato.ac.nz
}

Submitted: Jun 3, 2009; Accepted: Sep 6, 2010; Published: Sep 22, 2010

Mathematics Subject Classification: 05C38 (05C51)

\begin{abstract}
In this paper we examine the problem of decomposing the lexicographic product of a cycle with an empty graph into cycles of uniform length. We determine necessary and sufficient conditions for a solution to this problem when the cycles are of odd length. We apply this result to find necessary and sufficient conditions to decompose a complete equipartite graph into cycles of uniform length, in the case that the length is both odd and short relative to the number of parts.
\end{abstract}

\section{Introduction}

Before we venture further, we remind the reader of some definitions. A complete equipartite graph $K_{n(m)}$ has its $n m$ vertices partitioned into $n$ parts, often referred to as partite sets, each of size $m$, and there is an edge between any two vertices in different partite sets, but no edge between any two vertices in the same partite set. The lexicographic product 
$G * H$ of graphs $G$ and $H$ is the graph with vertex set $V(G) \times V(H)$, and with an edge joining $\left(g_{1}, h_{1}\right)$ to $\left(g_{2}, h_{2}\right)$ if and only if: $g_{1}$ is adjacent to $g_{2}$ in $G$; or $g_{1}=g_{2}$ and $h_{1}$ is adjacent to $h_{2}$ in $H$. Observe that $K_{n(m)}$ is isomorphic to $K_{n} * \bar{K}_{m}$.

We frequently exploit the following elementary facts abouts the lexicographic product. For any graph $G,\left(G * \bar{K}_{m}\right) * \bar{K}_{\ell} \cong G * \bar{K}_{m \ell}$. Also, if $G$ has an edge-disjoint decomposition into subgraphs $G_{1}, G_{2}, \ldots, G_{t}$, then $G * \bar{K}_{m}$ has an edge-disjoint decomposition into subgraphs $G_{1} * \bar{K}_{m}, G_{2} * \bar{K}_{m}, \ldots, G_{t} * \bar{K}_{m}$.

With the above observations, it should come as no surprise that results on decompositions of lexicographic products can be very useful tools in finding decompositions of complete equipartite graphs. This is the key approach in many papers, including: $[2,3,6]$ and indeed this paper.

Much work on cycle decompositions of $K_{n} * \bar{K}_{m}$ focuses on small values of $m, n$ or on small fixed cycle lengths. The problem of determining necessary and sufficient conditions for existence of an edge-disjoint decomposition of a complete graph $K_{n}$ ( $n$ odd) into $k$ cycles was finally completed in [1] and [11]. This graph can be regarded as a complete equipartite graph in which all the parts have size 1 . The same cycle decomposition problem for the graph $K_{n}-F$ where $F$ is a 1-factor and $n$ is even, was also solved in these papers. This latter graph can be regarded as a complete equipartite graph with $n / 2$ parts of size 2. These results are summarized in Theorem 7 in the next section.

As part of a more general result, Sotteau [14] showed that when $n=2, K_{n} * \bar{K}_{m}$ decomposes into cycles of length $k$ if and only if $k$ is even (as odd cycles are tripartite), $m$ is even, $k<2 m$ and $k$ divides $m^{2}$. The equivalent problem for $n=3$ has also been solved ([6]), and recently all the cases $n \leqslant 5$ were completed $([2,3])$.

Some work has also been done on cycle decompositions of $K_{n} * \bar{K}_{m}$ when the cycle length is small and specified (see [7] for small even length and arbitrary part sizes), of prime length $([10])$, and of length twice or thrice a prime $[12,13]$. Liu $([8,9])$ deals with resolvable cycle decompositions of complete equipartite graphs with any number of parts; the resolvability of course means a greater restriction on possible cycle lengths.

The results from this paper allow us to solve a significant chunk of the general problem of determining necessary and sufficient conditions for existence of an edge-disjoint decomposition of a complete equipartite graph into cycles of uniform length. In particular we solve all cases where the cycle length is odd and small relative to the number of parts.

Our main results are the following:

Theorem 1. The graph $C_{n} * \bar{K}_{m}$ decomposes into cycles of odd length $k$ if and only if:

- $n$ is odd,

- $n \leqslant k \leqslant m n$ and

- $k$ divides $\mathrm{nm}^{2}$.

Theorem 2. Let $n, m$ and $k$ be positive integers such that $m$ is odd, $k$ is odd and $3 \leqslant k \leqslant n$. Then the complete equipartite graph $K_{n} * \bar{K}_{m}$ admits a decomposition into cycles of length $k$ if and only if $n$ is odd and $k$ divides $m^{2}\left(\begin{array}{l}n \\ 2\end{array}\right)$. 
Theorem 3. Let $n, m$ and $k$ be positive integers such that $m$ is even, $k$ is odd and $3 \leqslant k \leqslant 2 n$. Then the complete equipartite graph $K_{n} * \bar{K}_{m}$ admits a decomposition into cycles of length $k$ if and only if $k$ divides $m^{2}\left(\begin{array}{l}n \\ 2\end{array}\right)$.

In Section 3 we show that Theorem 1, together with some known results from the literature given in Section 2, implies Theorems 2 and 3. This motivates the proof of Theorem 1 in Section 5, using preliminary lemmas from Section 4.

\section{Known Results}

The following known results should not be regarded as a comprehensive survey of results in the area (see the previous section); we list only the results relied upon in this paper.

A proof of the following result may be found in [6]:

Theorem 4. [Cavenagh] For $k \geqslant 3$ and $m \geqslant 1$, the graph $C_{k} * \bar{K}_{m}$

(i) has a decomposition into cycles of length $k$;

(ii) has a decomposition into cycles of length $\mathrm{km}$.

We sometimes need a more explicit version of Theorem 4 (ii).

Theorem 5. [Cavenagh] For $k \geqslant 3, m \geqslant 1$ and $m \notin\{2,6\}$, the graph $C_{k} * \bar{K}_{m}$ decomposes into cycles of length $\mathrm{km}$ in such a way that each cycle consists of a perfect matching between each pair of consecutive partite sets in $C_{k} * \bar{K}_{m}$.

The proof of Theorem 5 follows from the existence of a pair of MOLS of order not equal to 2 or 6 ; see, for example, the detail of Theorem 2.4 in [6].

It is also possible, under certain conditions, to take lexicographic products of closed trails (connected, even graphs) with empty graphs, and decompose such graphs into cycles. Some of these sufficient conditions are outlined in the following theorem, a special case of Theorem 1.1 from [13].

Theorem 6. [Smith] Let CT be a tripartite closed trail on $k$ edges having maximum degree $\Delta$. Then for all $m \geqslant \Delta / 2$, the graph $C T * \bar{K}_{m}$ can be decomposed into cycles of length $k$.

For the sake of brevity we define, for each positive integer $n$, the graph $G_{n}$ to be the complete graph $K_{n}$ when $n$ is odd, and the complete graph minus a 1-factor $K_{n}-F$ when $n$ is even. Hence $G_{n}$ is an even graph and $\left|E\left(G_{n}\right)\right|=n\left\lfloor\frac{n-1}{2}\right\rfloor$.

Necessary and sufficient conditions for decomposing $G_{n}$ into cycles of uniform length have been completely determined $([1,11])$ :

Theorem 7. [Alspach, Gavlas, Šajna] For each $k, n \geqslant 3$, the graph $G_{n}$ decomposes into cycles of length $k$ if and only if $k \leqslant n$ and $k$ divides $n\left\lfloor\frac{n-1}{2}\right\rfloor$. 
Necessary and sufficient conditions for decomposing $G_{n}$ into cycles of non-uniform length (the so-called Alspach Conjecture) have not been found in generality. However the following results from [4] and [5] will be particularly useful in this paper.

Theorem 8. [Bryant, Horsley] Let $n$ be a positive integer and let $k_{1}, k_{2}, \ldots, k_{x}$ be a list of integers such that

- $\left\lceil\frac{n}{2}\right\rceil \geqslant k_{1} \geqslant k_{2} \geqslant \cdots \geqslant k_{x} \geqslant 3 ;$

- $k_{1}+k_{2}+\cdots+k_{x}=n\left\lfloor\frac{n-1}{2}\right\rfloor ;$ and

- $2 k_{2} \geqslant k_{1}$.

Then there exists a decomposition of $G_{n}$ into $x$ cycles of lengths $k_{1}, k_{2}, \ldots, k_{x}$.

Theorem 9. [Bryant, Horsley] Let $n$ be a positive integer and let $k_{1}, k_{2}, \ldots, k_{x}$ be a list of integers such that

- $n \geqslant k_{1} \geqslant k_{2} \geqslant \cdots \geqslant k_{x} \geqslant\left\lceil\frac{n+4}{2}\right\rceil ;$ and

- $k_{1}+k_{2}+\cdots+k_{x}=n\left\lfloor\frac{n-1}{2}\right\rfloor$.

Then there exists a decomposition of $G_{n}$ into $x$ cycles of lengths $k_{1}, k_{2}, \ldots, k_{x}$.

The following is shown in [6].

Theorem 10. [Cavenagh] The graph $K_{3} * \bar{K}_{m}$ decomposes into cycles of length $k$ if and only if $k \leqslant 3 m$ and $k$ divides $3 m^{2}$.

Theorem 11. Let $k$ be odd and suppose that $k$ divides $m n, m(n-1)$ is even and $n \geqslant 3$. Then the graph $K_{n} * \bar{K}_{m}$ decomposes into cycles of length $k$.

Proof. When $n=3$, the result follows from Theorem 10. The graph $K_{6} * \bar{K}_{2} \cong G_{12}$ decomposes into 3 -cycles by Theorem 7 . The remaining cases follow from Liu [9].

\section{Decompositions of equipartite graphs}

In this section we show that Theorem 1, together with some known results from the literature, implies Theorems 2 and 3. The remainder of the paper is then devoted to proving Theorem 1. We first use Theorems 8 and 9 to prove the following preliminary lemma.

Lemma 1. Let $n, a$ and $s$ be positive integers such that $s$ is odd, $s \geqslant 3, n \geqslant a s^{2}$ and $a\left\lfloor n\left\lfloor\frac{n-1}{2}\right\rfloor\right.$. Then, for some integer $x$, there exists a decomposition of $G_{n}$ into $x$ cycles of lengths $a c_{1}, a c_{2}, \ldots, a c_{x}$ where each $c_{i}$ is odd and in the range $s \leqslant c_{i} \leqslant s^{2}$. 
Proof. Let $n^{\prime}=n\left\lfloor\frac{n-1}{2}\right\rfloor / a$. We write $n^{\prime}=s d+r$ where $d$ and $r$ are positive integers with $0 \leqslant r<s$. We now split the problem according to the parity of $r$.

Case 1. Suppose $r$ is even. If $a(s+r) \leqslant\left\lceil\frac{n}{2}\right\rceil$ we set $x=d$ and define

$$
c_{i}= \begin{cases}s+r, & \text { for } i=1 \\ s, & \text { for } 2 \leqslant i \leqslant x .\end{cases}
$$

Hence $a c_{1}+a c_{2}+\cdots+a c_{x}=n\left\lfloor\frac{n-1}{2}\right\rfloor$ and $2 a c_{2} \geqslant a c_{1} \geqslant a c_{2} \geqslant \cdots \geqslant a c_{x}$. The result then follows by Theorem 8. Suppose then that $a(s+r)>\left\lceil\frac{n}{2}\right\rceil$.

Since $a(s+r) \leqslant 2 a s \leqslant \frac{2 n}{s}$ we must have $s=3$ and hence $r=0$ or 2 . In fact, since $a s \leqslant \frac{n}{s}<\left\lceil\frac{n}{2}\right\rceil$ we must have $r=2$. Moreover, since $n \geqslant 9$ and $a(s+r)=5 a>\left\lceil\frac{n}{2}\right\rceil$ we have $a \neq 1$. Hence $n \geqslant a s^{2} \geqslant 18$ and $n^{\prime}=n\left\lfloor\frac{n-1}{2}\right\rfloor / a \geqslant s^{2}\left\lfloor\frac{n-1}{2}\right\rfloor \geqslant 9(8)$. Thus it is clear that there exist nonnegative integers $\alpha$ and $\beta$ such that $n^{\prime}=9 \alpha+7 \beta$. We set $x=\alpha+\beta$ and define

$$
c_{i}= \begin{cases}9, & \text { for } 1 \leqslant i \leqslant \alpha ; \\ 7, & \text { for } \alpha+1 \leqslant i \leqslant x .\end{cases}
$$

Hence $a c_{1}+a c_{2}+\cdots+a c_{x}=n\left\lfloor\frac{n-1}{2}\right\rfloor$ and $a c_{1} \geqslant a c_{2} \geqslant \cdots \geqslant a c_{x} \geqslant\left\lceil\frac{n+4}{2}\right\rceil$. The result then follows by Theorem 9 .

Case 2. Suppose $r$ is odd. We split the problem according to whether $r$ and $s$ are congruent modulo 4.

Case 2A. Suppose $r \not \equiv s(\bmod 4)$. If $\frac{a(3 s+r)}{2} \leqslant\left\lceil\frac{n}{2}\right\rceil$ we set $x=d-1$ and define

$$
c_{i}= \begin{cases}\frac{3 s+r}{2}, & \text { for } 1 \leqslant i \leqslant 2 ; \\ s, & \text { for } 3 \leqslant i \leqslant x .\end{cases}
$$

Hence $a c_{1}+a c_{2}+\cdots+a c_{x}=n\left\lfloor\frac{n-1}{2}\right\rfloor$ and $a c_{1}=a c_{2} \geqslant \cdots \geqslant a c_{x}$. The result then follows by Theorem 8. Suppose then that $\frac{a(3 s+r)}{2}>\left\lceil\frac{n}{2}\right\rceil$.

Since $\frac{a(3 s+r)}{2}<2 a s \leqslant \frac{2 n}{s}$ we must have $s=3$ and hence $r=1$. Moreover, since $\frac{a(3 s+r)}{2}=5 a>\left\lceil\frac{n}{2}\right\rceil$ and $n \geqslant 9$ we must have $a \neq 1$. Hence $n \geqslant a s^{2} \geqslant 18$ and $n^{\prime}=$ $n\left\lfloor\frac{n-1}{2}\right\rfloor / a \geqslant s^{2}\left\lfloor\frac{n-1}{2}\right\rfloor \geqslant 9(8)$. Thus it is clear that there exist nonnegative integers $\alpha$ and $\beta$ such that $n^{\prime}=9 \alpha+7 \beta$. We set $x=\alpha+\beta$ and define

$$
c_{i}= \begin{cases}9, & \text { for } 1 \leqslant i \leqslant \alpha ; \\ 7, & \text { for } \alpha+1 \leqslant i \leqslant x .\end{cases}
$$

Hence $a c_{1}+a c_{2}+\cdots+a c_{x}=n\left\lfloor\frac{n-1}{2}\right\rfloor$ and $a c_{1} \geqslant a c_{2} \geqslant \cdots \geqslant a c_{x} \geqslant\left\lceil\frac{n+4}{2}\right\rceil$. The result then follows by Theorem 9 .

Case 2B. Suppose $r \equiv s(\bmod 4)$. Hence $s \geqslant 5$ and $\frac{a(3 s+r+2)}{2}<a(2 s+1)<\frac{a s^{2}}{2} \leqslant\left\lceil\frac{n}{2}\right\rceil$. We set $x=d-1$ and define

$$
c_{i}= \begin{cases}\frac{3 s+r+2}{2}, & \text { for } i=1 \\ \frac{3 s+r-2}{2}, & \text { for } i=2 ; \\ s, & \text { for } 3 \leqslant i \leqslant x .\end{cases}
$$


Hence $a c_{1}+a c_{2}+\cdots+a c_{x}=n\left\lfloor\frac{n-1}{2}\right\rfloor$ and $2 a c_{2} \geqslant a c_{1} \geqslant a c_{2} \geqslant \cdots \geqslant a c_{x}$. The result then follows by Theorem 8 .

We are now ready to show that Theorem 1 implies Theorems 2 and 3.

\section{Proof of Theorem 2.}

The necessity of the conditions is obvious and hence we need only prove the sufficiency.

We write $k=a s^{2} t$ where $a\left|\left(\begin{array}{l}n \\ 2\end{array}\right), s t\right| m$ and $t$ is square free. Note that each of $a, s$ and $t$ are odd. If $s=a=1$ the result follows by Theorem 11, while if $s=1$ and $a \geqslant 3$ the result follows by applying Theorem 4 to an $a$-cycle decomposition of $K_{n}$ (Theorem 7). Hence we may assume that $s \geqslant 3$.

We need only show that $K_{n} * \bar{K}_{s}$ admits a decomposition into cycles of length $a s^{2}$ and the result then follows by Theorem 4 . Since $C_{a c} * \bar{K}_{s}$ admits a decomposition into cycles of length $a s^{2}$ for each odd $c$ in the range $s \leqslant c \leqslant s^{2}$ (see Theorem 1), it suffices to show that, for some integer $x, K_{n}$ can be decomposed into $x$ cycles of lengths $a c_{1}, a c_{2}, \ldots, a c_{x}$, where each $c_{i}$ is odd and in the range $s \leqslant c_{i} \leqslant s^{2}$. This follows from Lemma 1 .

\section{Proof of Theorem 3.}

The necessity of the conditions is obvious and hence we need only prove the sufficiency.

We write $k=a s^{2} t$ where $a\left|4\left(\begin{array}{c}n \\ 2\end{array}\right), 2 s t\right| m$ and $t$ is square free. Note that each of $a, s$ and $t$ are odd. If $s=a=1$ the result follows by Theorem 11, while if $s=1$ and $a \geqslant 3$ the result follows by applying Theorem 4 to an $a$-cycle decomposition of $K_{n} * \bar{K}_{2}$ (Theorem 7 ). Hence we may assume that $s \geqslant 3$.

We need only show that $K_{n} * \bar{K}_{2 s}$ admits a decomposition into cycles of length $a s^{2}$ and the result then follows by Theorem 4 . Since $C_{a c} * \bar{K}_{s}$ admits a decomposition into cycles of length $a s^{2}$ for each odd $c$ in the range $s \leqslant c \leqslant s^{2}$ (see Theorem 1), it suffices to show that, for some integer $x, K_{n} * \bar{K}_{2}$ can be decomposed into $x$ cycles of lengths $a c_{1}, a c_{2}, \ldots, a c_{x}$, where each $c_{i}$ is odd and in the range $s \leqslant c_{i} \leqslant s^{2}$. This follows from Lemma 1, noting that $K_{n} * \bar{K}_{2} \cong G_{2 n}$ and $4\left(\begin{array}{l}n \\ 2\end{array}\right)=2 n\left\lfloor\frac{2 n-1}{2}\right\rfloor$.

\section{Notation and preliminary lemmas}

In this section we introduce some notation, including the idea of weak and strong paths. These are the building blocks for our constructions in the next section.

In this section, $s$ and $\ell$ are positive integers. For $u \geqslant 1$, let $V_{u}=\left\{u_{0}, u_{1}, \ldots, u_{s-1}\right\}$. Let $L_{\ell}$ be the path of length $\ell$ (that is, with $\ell$ edges). We label the vertices of $L_{\ell} * \bar{K}_{s}$ with the elements of $\bigcup_{u \in \mathbb{Z}_{\ell+1}} V_{u}$, with an edge joining $u_{i}$ and $v_{j}$ if and only if $u$ and $v$ differ by 1 . We label the vertices of $C_{\ell} * \bar{K}_{s}$ with the elements of $\bigcup_{u \in \mathbb{Z}_{\ell}} V_{u}$, with an edge joining $u_{i}$ and $v_{j}$ if and only if $u$ and $v$ differ by $1(\bmod \ell)$. In general, we calculate the subscripts of vertex labels mod $s$. For $C_{\ell} * \bar{K}_{s}$ we calculate vertex labels mod $\ell$.

For a particular graph $G$ in $L_{\ell} * \bar{K}_{s}$ and integer $i$, we let $G \oplus i$ be the graph created from $G$ by adding $i$ to each vertex label of $G$, keeping subscripts fixed. We note that $G \oplus i$ 
is a subgraph of $L_{\ell+j} * \bar{K}_{s}$ for any $j \geqslant i$. We also define $G^{i}$ to be the graph created from $G$ by adding $i(\bmod s)$ to each subscript of each vertex of $G$. We similarly define graphs $G \oplus i$ and $G^{i}$ when $G$ is a particular subgraph in $C_{\ell} * \bar{K}_{s}$, this time calculating vertex labels $\bmod \ell$.

We consider an edge of the form $\left\{j_{i},(j+1)_{i+d}\right\}$ to have difference $d$ and type $(i, d)$. For any set $D \subseteq\{0,1, \ldots, s-1\}$ we define a weak $(\ell, s, D)$ - path to be any path in $L_{\ell} * \bar{K}_{s}$ with end vertices $0_{0}$ and $\ell_{0}$ which contains exactly one edge of each difference $d \in D$. We note that if $P$ is a weak $(\ell, s, D)$-path then $P$ has length $|D|$.

For any set $D \subseteq\{0,1, \ldots, s-1\}$ we define a strong $(\ell, s, D)$ - path to be any path in $L_{\ell} * \bar{K}_{s}$ with end vertices $0_{0}$ and $\ell_{0}$ which contains, for each $d \in D$ and each $i \in$ $\{0,1, \ldots, s-1\}$, exactly one edge of type $(i, d)$. We note that if $P$ is a strong $(\ell, s, D)$-path then $P$ has length $s|D|$.

Furthermore, we define a weak (respectively, strong) $(\ell, s, D)^{*}$-path to be a weak (respectively, strong) $(\ell, s, D)$-path such that for each $i \in\{1,2, \ldots, s-1\}$, at most one of the vertices in the set $\left\{0_{i}, \ell_{i}\right\}$ is included in the path.

Lemma 2. Let $\ell, s$ and a be positive integers. If there exists a weak $\left(\ell, s, \mathbb{Z}_{s}\right)^{*}$-path then there exists a decomposition of $C_{a \ell} * \bar{K}_{s}$ into cycles of length as.

Proof. Let $P$ be a weak $\left(\ell, s, \mathbb{Z}_{s}\right)^{*}$-path. Let $P^{\prime}$ be the path, in $L_{a \ell} * \bar{K}_{s}$, obtained by concatenating the paths $P, P \oplus \ell, P \oplus 2 \ell, \ldots, P \oplus(a-1) \ell$. Thus $P^{\prime}$ is a path of length as with end vertices $0_{0}$ and $(a \ell)_{0}$. Moreover, for each $d \in \mathbb{Z}_{s}$ and each $j \in\{0,1, \ldots, a-1\}, P^{\prime}$ contains exactly one edge of the form $\left\{(v+j \ell)_{i},(v+j \ell+1)_{i+d}\right\}$ for some $v \in\{0,1, \ldots, \ell-1\}$ and some $i \in\{0,1, \ldots, s-1\}$.

Let $C_{a \ell} * \bar{K}_{s}$ be the graph formed by identifying, for each $i \in \mathbb{Z}_{s}$, the vertices $0_{i}$ and $(a \ell)_{i}$ in the graph $L_{a \ell} * \bar{K}_{s}$, and $C$ be the subgraph of $C_{a \ell} * \bar{K}_{s}$ obtained by the same identification of vertices in $P^{\prime}$. Then $C$ is a cycle of length as. Moreover, the cycles of the form $C^{x} \oplus y$, where $0 \leqslant x \leqslant s-1$ and $0 \leqslant y \leqslant \ell-1$, decompose $C_{a \ell} * \bar{K}_{s}$ as required.

Lemma 3. Let $\ell, s$ and $a$ be positive integers. If there exists a strong $\left(\ell, s, \mathbb{Z}_{s}\right)^{*}$-path then there exists a decomposition of $C_{a \ell} * \bar{K}_{s}$ into cycles of length as ${ }^{2}$.

Proof. Let $P$ be a strong $\left(\ell, s, \mathbb{Z}_{s}\right)^{*}$-path. Let $P^{\prime}$ be the path, in $L_{a \ell} * \bar{K}_{s}$, obtained by concatenating the paths $P, P \oplus \ell, P \oplus 2 \ell, \ldots, P \oplus(a-1) \ell$. Thus $P^{\prime}$ is a path of length $a s^{2}$ with end vertices $0_{0}$ and $(a \ell)_{0}$. Moreover, for each $d \in \mathbb{Z}_{s}$, each $i \in \mathbb{Z}_{s}$ and each $j \in\{0,1, \ldots, a-1\}, P^{\prime}$ contains exactly one edge of the form $\left\{(v+j \ell)_{i},(v+j \ell+1)_{i+d}\right\}$ for some $v \in\{0,1, \ldots, \ell-1\}$.

Let $C_{a \ell} * \bar{K}_{s}$ be the graph formed by identifying, for each $i \in \mathbb{Z}_{s}$, the vertices $0_{i}$ and $(a \ell)_{i}$ in the graph $L_{a \ell} * \bar{K}_{s}$, and $C$ be the subgraph of $C_{a \ell} * \bar{K}_{s}$ obtained by the same identification of vertices in $P^{\prime}$. Then $P^{\prime}$ is a cycle of length $a s^{2}$. Moreover, the cycles $C, C \oplus 1, C \oplus 2, \ldots, C \oplus(\ell-1)$ decompose $C_{a \ell} * \bar{K}_{s}$ as required.

Lemma 4. There exists a weak $\left(\ell, t, \mathbb{Z}_{t}\right)^{*}$-path for any odd integers $t$ and $\ell$ such that $3 \leqslant \ell \leqslant t$. Moreover, there is such a path which uses no vertices of the form $0_{i}$ where $(t+1) / 2 \leqslant i \leqslant t-1$. 
Proof. Let $u=t+1-\ell$. Note that $1 \leqslant u$. Also, since $\ell$ and $t$ are odd, $u$ is also odd. Let $u=2 U+1$. We will form the required path by concatenating two paths: $P_{1}$ (of length $u$ ) and $P_{2}$ (of length $t-u$ ). Note that $t-u>1$ and $t-u$ is even. If $u=1$, then $P_{1}=\left[0_{0}, 1_{0}\right]$. Otherwise, $P_{1}=\left[0_{0}, 1_{U}, 0_{1}, 1_{U-1}, \ldots, 0_{U}, 1_{0}\right]$. Next, $P_{2}=$ $\left[1_{0}, 2_{U+1}, 3_{0}, 4_{U+2}, 5_{0}, \ldots,(t-u-1)_{0},(t-u)_{(t-1) / 2},(t-u+1)_{0}\right]$.

Before we prove Lemma 8, we need the following preliminary lemmas.

Lemma 5. There exists a strong $\left(2 s, s,\left\{d, d^{\prime}\right\}\right)^{*}$-path for any integers $s, d$ and $d^{\prime}$ such that $1 \leqslant d, d^{\prime} \leqslant s$ and $\operatorname{gcd}\left(d, d^{\prime}\right)=1$.

Proof. Let $\operatorname{gcd}(d, s)=g$ and $\operatorname{gcd}\left(d^{\prime}, s\right)=h$. Since $\operatorname{gcd}\left(d, d^{\prime}\right)=1$, we have that $\operatorname{gcd}(g, h)=$ 1 and consequently $g h \leqslant s$. Let $s=h h^{\prime}=g g^{\prime}$. Note that $h \leqslant s / g=g^{\prime}$ and similarly $g \leqslant h^{\prime}$.

To describe our strong $\left(2 s, s,\left\{d, d^{\prime}\right\}\right)^{*}$-path, we list the subscripts of the vertices (calculated $\bmod s$ as usual) in a sequence $S$, so that $a$ is the $i$ th element of $S$ if and only if $(i-1)_{a}$ is the $i$ th vertex in our path.

Firstly, suppose that $h^{\prime}=1$. Then $s=h$ which implies that $d^{\prime}=s$, so that $g=\operatorname{gcd}(d, s)=\operatorname{gcd}\left(d, d^{\prime}\right)=1$ and $g^{\prime}=s$. In this case, our sequence $S$ is given by:

$$
0,0, d, d, 2 d, 2 d, \ldots,(s-1) d,(s-1) d, 0 .
$$

The case $g^{\prime}=1$ is similar.

Henceforth we may assume that $h^{\prime}>1$ and $g^{\prime}>1$. For each $a$ such that $1 \leqslant a \leqslant h-1$ we define $P_{a}$ to be the sequence

$$
a d, a d+d^{\prime}, a d+2 d^{\prime}, \ldots a d+\left(h^{\prime}-1\right) d^{\prime}, a d .
$$

For each $b$ such that $1 \leqslant b \leqslant g-1$ we define $Q_{b}$ to be the sequence

$$
b d^{\prime}, b d^{\prime}+d, b d^{\prime}+2 d, \ldots b d^{\prime}+\left(g^{\prime}-1\right) d, b d^{\prime} .
$$

If $h=g=1$, the sequence $S$ is given by:

$$
0, d, 2 d, \ldots,(s-1) d, 0, d^{\prime}, 2 d^{\prime}, \ldots,(s-1) d^{\prime}, 0 .
$$

If $h=1$ and $g>1$, our sequence $S$ is:

$$
0, d, 2 d, \ldots,\left(g^{\prime}-1\right) d, 0, Q_{1}, Q_{2}, \ldots, Q_{g-1}, g d^{\prime},(g+1) d^{\prime},(s-1) d^{\prime}, 0 .
$$

If $h>1$ and $g=1, S$ is given by

$$
0, P_{1}, P_{2}, \ldots, P_{h-1}, h d,(h+1) d, \ldots,(s-1) d, 0, d^{\prime}, 2 d^{\prime}, \ldots,\left(h^{\prime}-1\right) d^{\prime}, 0 .
$$

Finally, we are left with the case $h>1$ and $g>1$. We then use the sequence $S$ :

$$
\begin{gathered}
0, P_{1}, P_{2}, \ldots, P_{h-1}, h d,(h+1) d, \ldots,\left(g^{\prime}-1\right) d, 0, Q_{1}, Q_{2}, \ldots \\
\ldots, Q_{g-1}, g d^{\prime},(g+1) d^{\prime}, \ldots,\left(h^{\prime}-1\right) d^{\prime}, 0 .
\end{gathered}
$$


Lemma 6. There exists a strong $(s, s,\{1,2, \ldots, a\})^{*}$-path for any odd integers a and $s$ such that $1 \leqslant a \leqslant s-2$.

Proof. Our aim is to construct a path $P$ of length $a$, in $L_{1} * \bar{K}_{s}$, with the following properties:

(i) $P$ has end vertices $0_{0}$ and $1_{x}$ where $1 \leqslant x \leqslant s-1$ and $\operatorname{gcd}(x, s)=1$;

(ii) $P$ contains exactly one edge of each difference $d \in\{1,2, \ldots, a\}$;

(iii) the graph formed by concatenating $P$ and $P^{x} \oplus 1$ is a path.

It is easy to see that the required strong $(s, s,\{1,2, \ldots, a\})^{*}$-path can then be obtained by concatenating the paths $P, P^{x} \oplus 1, P^{2 x} \oplus 2, \ldots, P^{(s-1) x} \oplus(s-1)$. We form $P$ as follows.

If $a=1$ or $a=3$, then $P=\left[0_{0}, 1_{1}\right]$ or $P=\left[0_{0}, 1_{2}, 0_{1}, 1_{4}\right]$ (respectively). Henceforth $a \geqslant 5$. If $a=4 A+1$,

$$
P=\left[0_{0}, 1_{1}, 0_{s-1}, 1_{2}, 0_{s-2}, \ldots, 1_{A}, 0_{s-A-1}, 1_{A+1}, 0_{s-A-2}, \ldots, 0_{s-2 A-1}, 1_{s-1}\right] .
$$

If $a=4 A+3=s-2$, then

$$
P=\left[0_{0}, 1_{1}, 0_{s-1}, 1_{2}, 0_{s-2}, \ldots, 1_{A}, 0_{s-A-1}, 1_{A+1}, 0_{s-A-2}, \ldots, 1_{2 A+1}, 0_{1}, 1_{s-1}\right] .
$$

Otherwise $a=4 A+3<s-2$ and

$$
P=\left[0_{0}, 1_{1}, 0_{s-1}, 1_{2}, 0_{s-2}, \ldots, 1_{A}, 0_{s-A-1}, 1_{A+1}, 0_{s-A-2}, \ldots, 0_{s-2 A-2}, 1_{s-2}\right] .
$$

In each case it is an easy exercise to check that $P$ satisfies conditions (i), (ii) and (iii) above. The result follows.

Lemma 7. Let $b$ be even, $s$ be odd and $2 \leqslant b \leqslant 2 s$. Then there exists a strong $(b, s,\{0, s-1\})$-path which includes the edge $\left[0_{0}, 1_{0}\right]$ but does not contain any of the vertices $0_{1}, 0_{2}, \ldots, 0_{s-1}$. Furthermore, if $b \geqslant 4$ there is such a path which does not contain any of the vertices $b_{1}, b_{2}, \ldots, b_{s-1}$.

Proof. The path

$$
P=\left[0_{0}, 1_{0}, 2_{s-1}, 1_{s-1}, 2_{s-2}, \ldots, 2_{b / 2-1}, 3_{b / 2-1}, 4_{b / 2-2}, 5_{b / 2-2}, \ldots, b_{0}\right]
$$

has the required properties.

Lemma 8. Let $c$ and $s$ be odd, $s \geqslant 3$ and $s+4 \leqslant c \leqslant s^{2}$. Then there exists a strong $\left(c, s, \mathbb{Z}_{s}\right)^{*}$-path which includes the edge $\left[0_{0}, 1_{0}\right]$. 
Proof. Write $c=a s+b$ where $a$ is odd, $b$ is even and $2 \leqslant b \leqslant 2 s$. Note that $1 \leqslant a \leqslant s-2$, and if $a=1$ then $b \geqslant 4$.

We set $x=(a-1) / 2$ and $A=s-1-a$. Partition $\mathbb{Z}_{s}$ into the sets $D=\{0, s-1\}$, $D^{\prime}=\{1,2, \ldots, A\}$ and, in the case $x \geqslant 1, D_{i}=\{A+2 i-1, A+2 i\}, i=1,2, \ldots, x$.

Then there exists a strong $(b, s, D)$-path, $P$ say, which includes the edge $\left[0_{0}, 1_{0}\right]$, by Lemma 7 , and a strong $\left(s, s, D^{\prime}\right)^{*}$-path, $P^{\prime}$ say, by Lemma 6 . Furthermore, if $x \geqslant 1$ there exists a strong $\left(2 s, s, D_{i}\right)$-path, $P_{i}$ say, for each $i=1,2, \ldots, x$, by Lemma 5 . The required strong $\left(c, s, \mathbb{Z}_{s}\right)^{*}$-path is then obtained by concatenating the paths

$$
P, P_{1} \oplus b, P_{2} \oplus(b+2 s), \ldots, P_{x} \oplus(b+(x-1) 2 s), P^{\prime} \oplus(b+2 x s)
$$

(omitting the paths $P_{1} \oplus b, P_{2} \oplus(b+2 s), \ldots, P_{x} \oplus(b+(x-1) 2 s)$ in the case $\left.x=0\right)$. It is easy to see that this does indeed form a strong $\left(c, s, \mathbb{Z}_{s}\right)^{*}$-path since none of the paths $P, P_{1}, P_{2}, \ldots, P_{x}$ contain any of the vertices $0_{1}, 0_{2}, \ldots, 0_{s-1}$, and furthermore, the path $P_{x}$ (respectively, $P$ in the case $x=0$ and $b \geqslant 4$ ) does not contain any of the vertices $(2 s)_{1},(2 s)_{2}, \ldots,(2 s)_{s-1}$ (respectively, $\left.b_{1}, b_{2}, \ldots, b_{s-1}\right)$.

We refer the reader to Figure 1 for a concrete example of this construction.

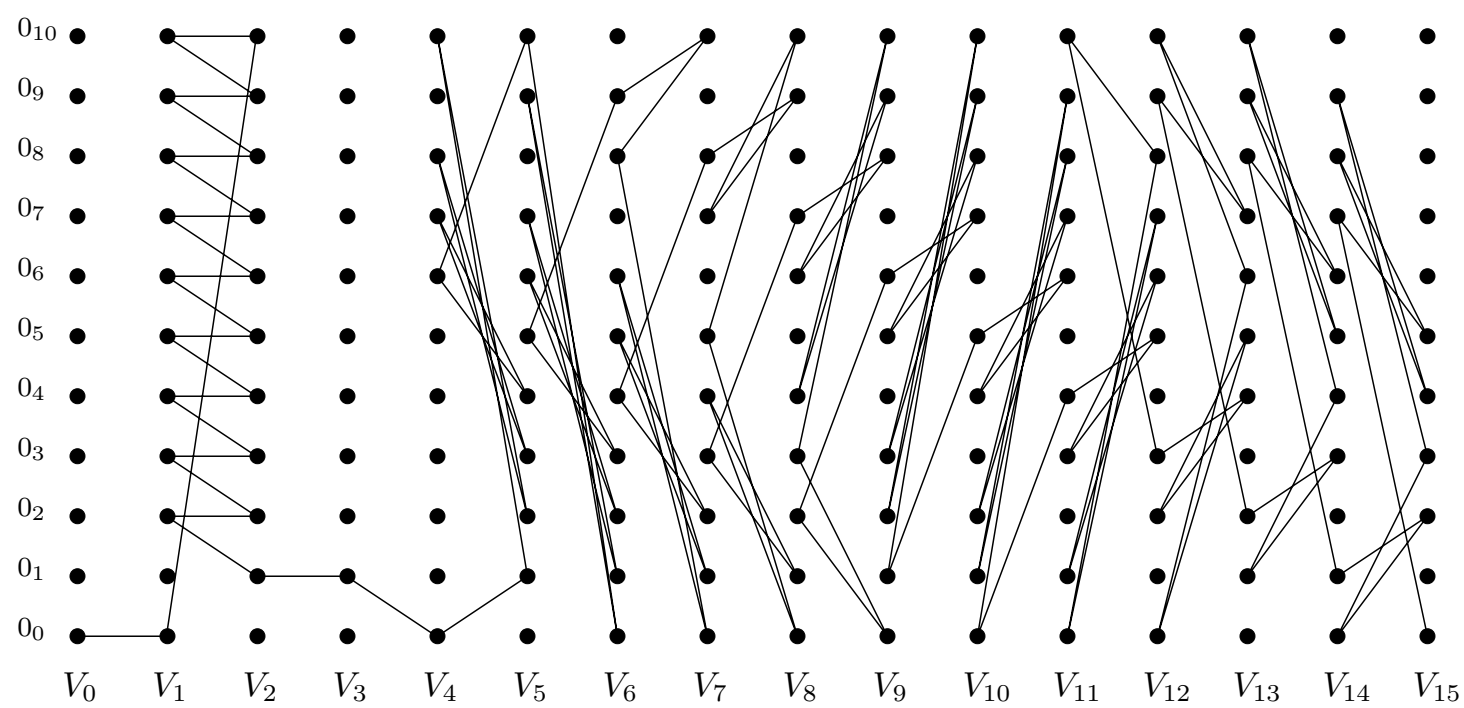

Figure 1: A strong $\left(15,11, \mathbb{Z}_{11}\right)^{*}$-path, constructed as in Lemma 8.

Unfortunately the techniques in the previous lemma fail if we try to construct a $(s+$ $\left.2, s, \mathbb{Z}_{s}\right)^{*}$-path in the same manner. To see this, $P^{\prime}$ and $P \oplus(2)$ defined as in the proof of the previous lemma do not combine to make a proper path. Instead we take a different approach. Admittedly some of the detail in the following lemma is fiddly. We encourage the reader to refer to Figures 2, 3, 4 and 5, which exhibit concrete examples.

Lemma 9. Let $s$ be odd and $s \geqslant 3$. Then there exists a strong $\left(s+2, s, \mathbb{Z}_{s}\right)^{*}$-path.

Proof. We split the proof into four cases, depending on the congruency of $s(\bmod 8)$. 
Case $1: s \equiv 3(\bmod 8)$. For $s=3$ a suitable $P$ is given by:

$$
\left[0_{0}, 1_{2}, 0_{2}, 1_{1}, 2_{1}, 3_{2}, 4_{0}, 3_{0}, 4_{1}, 5_{0}\right] .
$$

Otherwise assume that $s \geqslant 11$.

For each $i, 0 \leqslant i \leqslant(s-7) / 4$, define the following path $P_{i}$ of length $4 s$ :

$$
\begin{aligned}
P_{i}= & {\left[(4 i)_{i},(4 i+1)_{(s-3) / 2-i},(4 i)_{i-1},(4 i+1)_{(s-5) / 2-i}, \ldots,(4 i)_{i+1},(4 i+1)_{(s-1) / 2-i},\right.} \\
& (4 i+2)_{(s-1) / 2-i},(4 i+3)_{i},(4 i+2)_{(s-3) / 2-i},(4 i+3)_{i-1}, \ldots,(4 i+2)_{(s+1) / 2-i}, \\
& \left.(4 i+3)_{i+1},(4 i+4)_{i+1}\right] .
\end{aligned}
$$

Note that $P_{i}$ includes an edge of type $(j, d)$ for each $j$ such that $0 \leqslant j \leqslant s-1$ and $d \in\{ \pm((s-1) / 2-2 i), \pm((s-3) / 2-2 i)\}$, except for $(j, d) \in\{(i,(s-1) / 2-2 i),((s-$ $1) / 2-i, 2 i-(s-3) / 2\}$. (Edges of the excluded types are later used in $R_{(s-19) / 8-\lfloor i / 2\rfloor}$ for $0 \leqslant i \leqslant(s-15) / 4$, or in $Q$ when $i \in\{(s-11) / 4,(s-7) / 4\}$.)

Let $P^{\prime}$ be the path of length $s^{2}-3 s$ formed by concatenating $P_{0}, P_{1}, \ldots, P_{(s-7) / 4}$. Observe that $P$ includes edges of type $(j, 0)$, where

$$
j \in\{1,2, \ldots,(s-3) / 4\} \cup\{(s+5) / 4,(s+9) / 4, \ldots,(s-1) / 2\} .
$$

Next, let $Q$ be the following path of length 15:

$$
\begin{aligned}
Q= & {\left[(s-3)_{(s-3) / 4},(s-2)_{(s+1) / 4},(s-3)_{(s+1) / 4},(s-2)_{(s+5) / 4},(s-1)_{(s+1) / 4},\right.} \\
& s_{(s-3) / 4},(s-1)_{(s+5) / 4}, s_{(s+9) / 4},(s+1)_{(s+5) / 4}, s_{(s-7) / 4},(s-1)_{(s-3) / 4}, \\
& \left.(s-2)_{(s-7) / 4},(s-3)_{(s+9) / 4},(s-2)_{(s+13) / 4},(s-1)_{(s+9) / 4},(s-2)_{(s-11) / 4}\right] .
\end{aligned}
$$

Next, for each $i, 0 \leqslant i \leqslant(s-19) / 8$, we define paths of length 12 :

$$
\begin{aligned}
R_{i}= & {\left[(s-2)_{(s-11) / 4-2 i},(s-1)_{(s-7) / 4-2 i},(s)_{(s-11) / 4-2 i},(s-1)_{(s+13) / 4+2 i},\right.} \\
& (s)_{(s+17) / 4+2 i},(s+1)_{(s+13) / 4+2 i},(s)_{(s-15) / 4-2 i},(s-1)_{(s-11) / 4-2 i}, \\
& (s-2)_{(s-15) / 4-2 i},(s-3)_{(s+17) / 4+2 i},(s-2)_{(s+21) / 4+2 i},(s-1)_{(s+17) / 4+2 i}, \\
& (s-2)_{(s-19) / 4-2 i} .
\end{aligned}
$$

(For $s=11$, no such paths are included in the final concatenation.)

Next, for each $i, 0 \leqslant i \leqslant(s-7) / 4$, we define paths:

$$
H_{i}=\left[(s-1)_{s-2 i-1},(s-2)_{s-2 i-1},(s-3)_{s-2 i-2},(s-2)_{s-2 i-2},(s-1)_{s-2 i-3}\right]
$$

and

$$
J_{i}=\left[s_{s-2 i-3},(s+1)_{s-2 i-2}, s_{s-2 i-1}\right] .
$$

Finally, we form the path $P$ of length $s^{2}$ by concatenating, in order: $P^{\prime}, Q, R_{0}$, $R_{1}, \ldots R_{(s-19) / 8},\left[(s-2)_{0},(s-1)_{s-1}\right], H_{0}, H_{1}, \ldots H_{(s-7) / 4},\left[(s-1)_{(s+1) / 2}, s_{(s+1) / 2}\right]$, $J_{(s-7) / 4}, J_{(s-11) / 4}, \ldots, J_{0},\left[s_{s-1},(s+1)_{0}, s_{0},(s+1)_{1},(s+2)_{0}\right]$. 


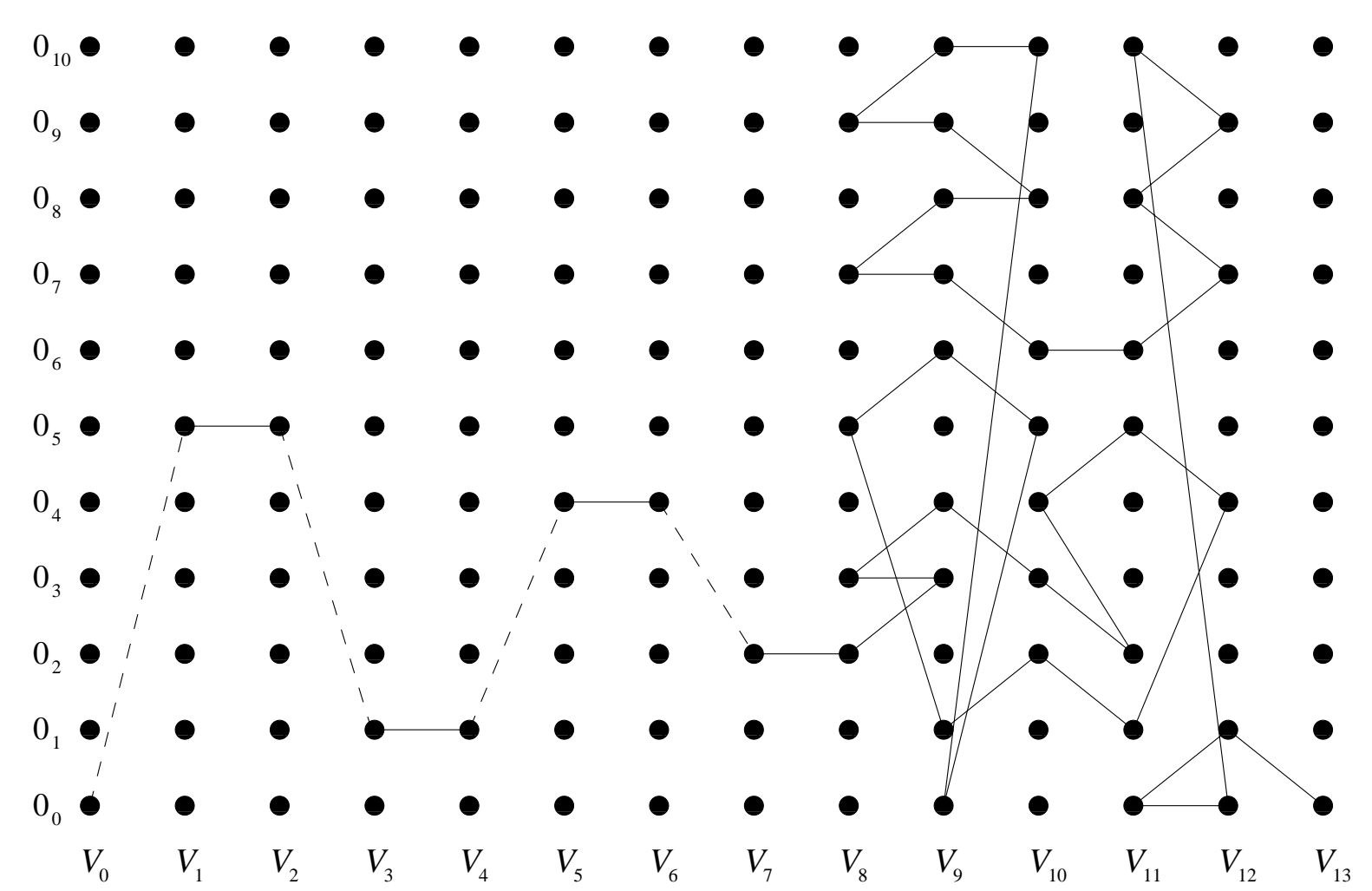

Figure 2: A strong $\left(13,11, \mathbb{Z}_{11}\right)^{*}$-path, constructed as in Case 1 of Lemma 9. For visual simplicty, we have used the following convention. A dashed line of type $(i, d)$ between partite sets of the form $V_{2 x}$ and $V_{2 x+1}$ means that we use all edges of difference $d$ and $d-1$ between those partite sets except for the dashed edge.

Case 2: $s \equiv 7(\bmod 8)$.

For each $i, 0 \leqslant i \leqslant(s-7) / 4$, define paths $P_{i}$ as in the previous case. Let $P^{\prime}$ be the path of length $s^{2}-3 s$ formed by concatenating $P_{0}, P_{1}, \ldots, P_{(s-7) / 4}$.

Next, let $Q$ be the following path:

$$
\begin{aligned}
Q= & {\left[(s-3)_{(s-3) / 4},(s-2)_{(s+1) / 4},(s-3)_{(s+1) / 4},(s-2)_{(s+5) / 4},(s-1)_{(s+1) / 4},\right.} \\
& s_{(s-3) / 4},(s-1)_{(s+5) / 4}, s_{(s+9) / 4},(s+1)_{(s+5) / 4}, s_{(s-7) / 4},(s-1)_{(s-3) / 4}, \\
& \left.(s-2)_{(s-7) / 4}\right]
\end{aligned}
$$

Next, for each $i, 0 \leqslant i \leqslant(s-15) / 8$, we define paths:

$$
\begin{aligned}
R_{i}= & {\left[(s-2)_{(s-7) / 4-2 i},(s-3)_{(s+9) / 4+2 i},(s-2)_{(s+13) / 4+2 i},(s-1)_{(s+9) / 4+2 i},\right.} \\
& (s-2)_{(s-11) / 4-2 i},(s-1)_{(s-7) / 4-2 i}, s_{(s-11) / 4-2 i},(s-1)_{(s+13) / 4+2 i}, \\
& s_{(s+17) / 4+2 i},(s+1)_{(s+13) / 4+2 i}, s_{(s-15) / 4-2 i},(s-1)_{(s-11) / 4-2 i}, \\
& \left.(s-2)_{(s-15) / 4-2 i}\right]
\end{aligned}
$$


Next, for each $i, 0 \leqslant i \leqslant(s-11) / 4$, we define paths:

$$
H_{i}=\left[s_{s-2 i-3},(s-1)_{s-2 i-3}, s_{s-2 i-2},(s+1)_{s-2 i-2}, s_{s-2 i-1}\right]
$$

and

$$
J_{i}=\left[(s-2)_{s-2 i-1},(s-3)_{s-2 i-2},(s-2)_{s-2 i-3}\right] .
$$

Finally, we form the path $P$ by concatenating, in order: $P^{\prime}, Q, R_{0}, R_{1}, \ldots R_{(s-15) / 8}$, $\left[(s-2)_{0},(s-1)_{s-1},(s-2)_{s-1}\right], J_{0}, J_{1}, \ldots J_{(s-11) / 4}$,

$\left[(s-2)_{(s+5) / 2},(s-3)_{(s+3) / 2},(s-2)_{(s+1) / 2},(s-1)_{(s+1) / 2}, s_{(s+3) / 2},(s+1)_{(s+3) / 2}, s_{(s+5) / 2}\right]$, $H_{(s-11) / 4}, H_{(s-15) / 4}, \ldots, H_{0},\left[s_{s-1},(s+1)_{0},(s+2)_{0}\right]$. (For the case $s=7$, all paths of the form $R_{i}, J_{i}$ and $H_{i}$ are omitted, in any case they are undefined.)

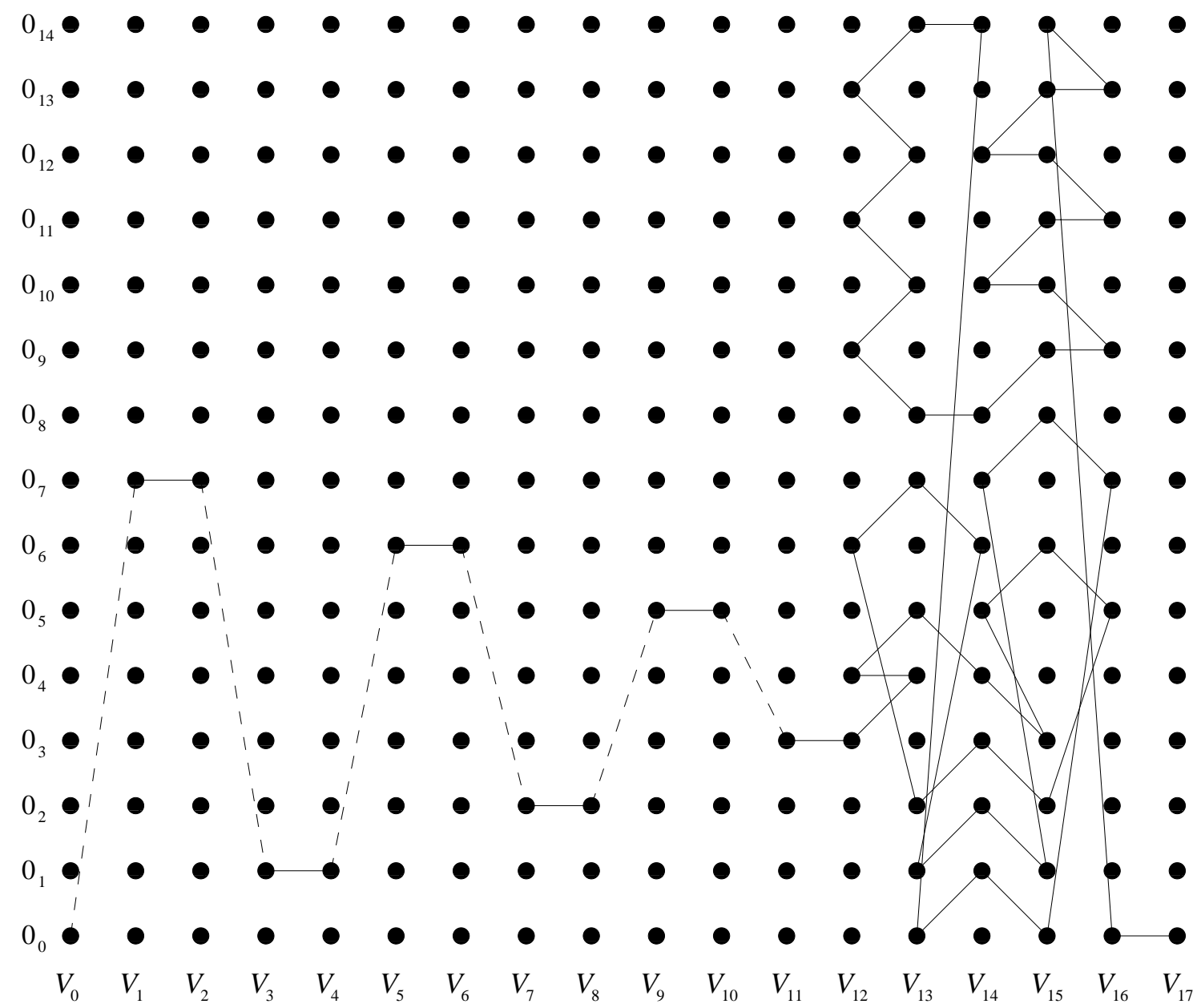

Figure 3: strong $\left(17,15, \mathbb{Z}_{15}\right)^{*}$-path, constructed as in Case 2 of Lemma 9. See Figure 2 for the meaning of dashed lines.

Case 3: $s \equiv 5(\bmod 8)$. For $s=5$ a suitable $P$ is given by:

$$
\left[0_{0}, 1_{2}, 0_{4}, 1_{1}, 0_{3}, 1_{0}, 2_{4}, 1_{4}, 2_{0}, 3_{3}, 2_{1}, 3_{4}, 2_{2}, 3_{0}, 4_{0}, 5_{1}, 4_{1}, 3_{2}, 4_{2}, 5_{3}, 4_{3}, 5_{4}, 6_{3}, 7_{2}, 6_{1}, 7_{0}\right] .
$$


Otherwise assume that $s \geqslant 13$.

For each $i, 0 \leqslant i \leqslant(s-9) / 4$, define paths $P_{i}$ of length $4 s$, as in the previous cases. Next, we define the following path $P^{\prime}$ of length $2 s-1$ :

$$
\begin{aligned}
P^{\prime}= & {\left[(s-5)_{(s-5) / 4},(s-4)_{(s-13) / 4},(s-5)_{(s-21) / 4},\right.} \\
& \left.(s-4)_{(s-29) / 4}, \ldots,(s-4)_{(s+3) / 4}\right] .
\end{aligned}
$$

Let $P^{\prime \prime}$ be the path of length $s^{2}-3 s-1$ which is a concatenation of $P_{0}, P_{1}, \ldots, P_{(s-9) / 4}$ and $P^{\prime}$. We next define $Q$ :

$$
\begin{aligned}
Q= & {\left[(s-4)_{(s+3) / 4},(s-3)_{(s+7) / 4},(s-2)_{(s+3) / 4},(s-3)_{(s+3) / 4},(s-2)_{(s-1) / 4},\right.} \\
& (s-3)_{(s-1) / 4},(s-2)_{(s-5) / 4},(s-1)_{(s-1) / 4}, s_{(s+3) / 4}, \\
& (s-1)_{(s-5) / 4}, s_{(s-9) / 4},(s+1)_{(s-5) / 4}, s_{(s+7) / 4}, \\
& \left.(s-1)_{(s+11) / 4},(s-2)_{(s+7) / 4},(s-3)_{(s-9) / 4},(s-2)_{(s-13) / 4},(s-1)_{(s-9) / 4}\right] .
\end{aligned}
$$

Next, for each $i, 0 \leqslant i \leqslant(s-21) / 8$, define:

$$
\begin{aligned}
R_{i}= & {\left[(s-1)_{(s-9) / 4-2 i},(s-2)_{(s+11) / 4+2 i},(s-1)_{(s+15) / 4+2 i}, s_{(s+11) / 4+2 i},\right.} \\
& (s-1)_{(s-13) / 4-2 i}, s_{(s-17) / 4-2 i},(s+1)_{(s-13) / 4-2 i}, s_{(s+15) / 4+2 i}, \\
& (s-1)_{(s+19) / 4+2 i},(s-2)_{(s+15) / 4+2 i},(s-3)_{(s-17) / 4-2 i},(s-2)_{(s-21) / 4-2 i}, \\
& \left.(s-1)_{(s-17) / 4-2 i}\right] .
\end{aligned}
$$

(For $s=13$, no such paths are included in the final concatenation.)

Next, for each $i, 0 \leqslant i \leqslant(s-9) / 4$, we define paths:

$$
H_{i}=\left[(s-2)_{s-2 i-1},(s-1)_{s-2 i-2},(s-2)_{s-2 i-2},(s-3)_{s-2 i-3},(s-2)_{s-2 i-3}\right]
$$

and

$$
J_{i}=\left[(s+1)_{s-2 i-3}, s_{s-2 i-2},(s+1)_{s-2 i-1}\right] .
$$

Finally, we form the path $P$ by concatenating, in order: $P^{\prime \prime}, Q, R_{0}, R_{1}, \ldots R_{(s-21) / 8}$, $\left[(s-1)_{1},(s-2)_{(s-1) / 2},(s-3)_{0},(s-2)_{s-1}\right], H_{0}, H_{1}, \ldots H_{(s-9) / 4},\left[(s-2)_{(s+3) / 2},(s-1)_{(s+1) / 2}\right.$, $\left.s_{(s-1) / 2},(s+1)_{(s+1) / 2}, s_{(s+1) / 2},(s+1)_{(s+3) / 2}\right], J_{(s-9) / 4}, J_{(s-13) / 4}, \ldots, J_{0},\left[(s+1)_{s-1}, s_{s-1},(s+\right.$ $\left.1)_{0},(s+2)_{0}\right]$.

Case 4: $s \equiv 1(\bmod 8)$.

The path $P^{\prime \prime}$ is defined exactly as in the previous case.

Next, we define the following path $Q$ :

$$
\begin{aligned}
Q= & {\left[(s-4)_{(s+3) / 4},(s-3)_{(s+3) / 4},(s-2)_{(s-1) / 4},(s-3)_{(s-1) / 4},\right.} \\
& (s-2)_{(s-5) / 4},(s-1)_{(s-1) / 4}, s_{(s+3) / 4},(s-1)_{(s-5) / 4}, \\
& s_{(s-9) / 4},(s+1)_{(s-5) / 4}, s_{(s+7) / 4},(s-1)_{(s+3) / 4}, \\
& \left.(s-2)_{(s+7) / 4},(s-3)_{(s-9) / 4}\right]
\end{aligned}
$$




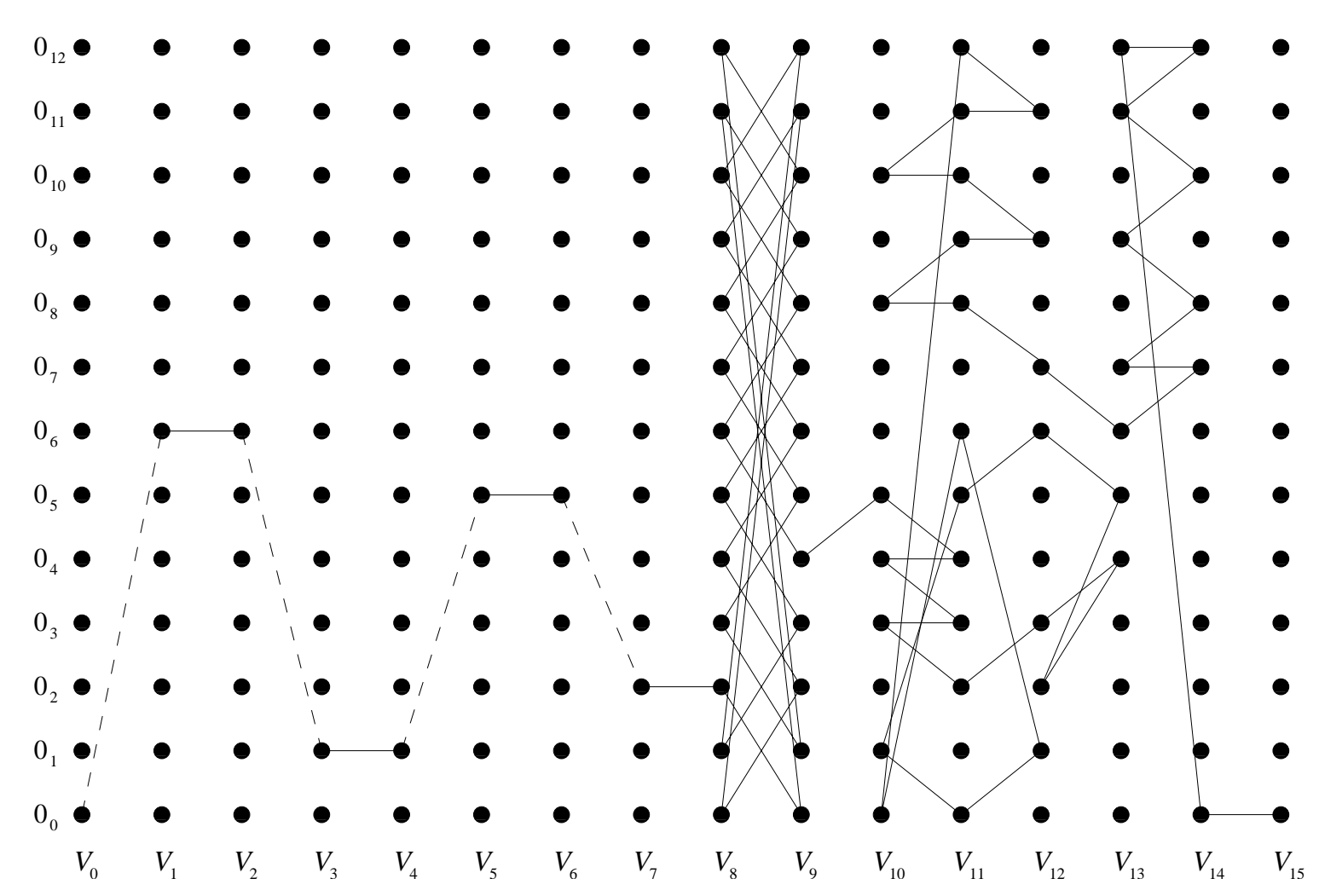

Figure 4: A strong $\left(15,13, \mathbb{Z}_{13}\right)^{*}$-path, constructed as in Case 3 of Lemma 9. See Figure 2 for the meaning of dashed lines.

Next, for each $i, 0 \leqslant i \leqslant(s-17) / 8$, define:

$$
\begin{aligned}
R_{i}= & {\left[(s-3)_{(s-9) / 4-2 i},(s-2)_{(s-13) / 4-2 i},(s-1)_{(s-9) / 4-2 i},(s-2)_{(s+11) / 4+2 i},\right.} \\
& (s-1)_{(s+7) / 4+2 i}, s_{(s+11) / 4+2 i},(s-1)_{(s-13) / 4-2 i}, s_{(s-17) / 4-2 i}, \\
& (s+1)_{(s-13) / 4-2 i}, s_{(s+15) / 4+2 i},(s-1)_{(s+11) / 4+2 i},(s-2)_{(s+15) / 4+2 i}, \\
& (s-3)_{(s-17) / 4-2 i} .
\end{aligned}
$$

(For $s=9$, no such paths are included in the final concatenation.)

Next, for each $i, 0 \leqslant i \leqslant(s-9) / 4$, we define paths:

$$
H_{i}=\left[(s-2)_{s-2 i-2},(s-3)_{s-2 i-3},(s-2)_{s-2 i-3},(s-1)_{s-2 i-4},(s-2)_{s-2 i-4}\right]
$$

and

$$
J_{i}=\left[s_{s-2 i-4},(s+1)_{s-2 i-3}, s_{s-2 i-2}\right] .
$$

Finally, we form $P$ by concatenating, in order: $P^{\prime \prime}, Q, R_{0}, R_{1}, \ldots R_{(s-17) / 8},\left[(s-3)_{0},(s-\right.$ $\left.2)_{s-1},(s-3)_{s-1},(s-2)_{s-2}\right], H_{0}, H_{1}, \ldots, H_{(s-9) / 4},\left[(s-2)_{(s+1) / 2},(s-1)_{(s-1) / 2}, s_{(s+1) / 2}\right]$, $J_{(s-9) / 4}, J_{(s-13) / 4}, \ldots, J_{0},\left[s_{s-2},(s-1)_{s-2}, s_{s-1},(s+1)_{0},(s+2)_{0}\right]$.

Combining Lemmas 9 and 8, we have the following. 


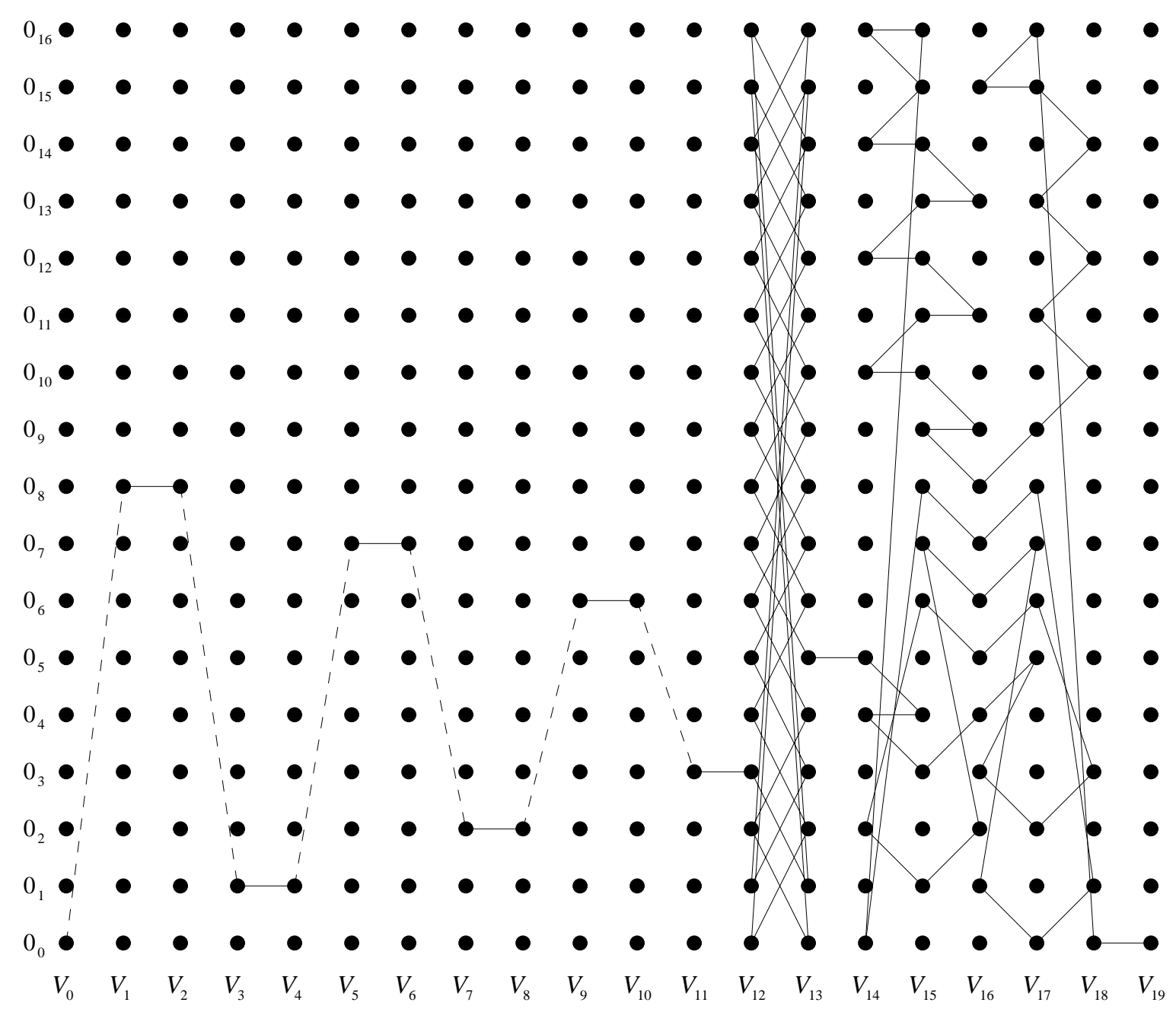

Figure 5: A strong $\left(19,17, \mathbb{Z}_{17}\right)^{*}$-path, constructed as in Case 4 of Lemma 9. See Figure 2 for the meaning of dashed lines.

Lemma 10. For each odd $c$ and $s$ such that $s<c \leqslant s^{2}$, there exists a strong $\left(c, s, \mathbb{Z}_{s}\right)^{*}$ path $P$.

\section{$5 \quad$ Lexicographic products of cycles and empty graphs}

In this section we examine the problem of decomposing $C_{k} * \bar{K}_{m}$ into cycles of arbitrary odd length $k$. The aim of this section is to prove Theorem 1.

Proof of Theorem 1.

We first do the easy part: justifying the necessity of these conditions. If $n$ is even, $C_{n} * \bar{K}_{m}$ is a bipartite graph, thus cannot contain any cycles of odd length. Thus $n$ must be odd. Moreover, any cycle in $C_{n} * \bar{K}_{m}$ of size less than $n$ must be bipartite, thus $n \leqslant k$. The number of vertices and edges of $C_{n} * \bar{K}_{m}$ are $n m$ and $n m^{2}$, respectively, hence the 
remaining conditions.

The rest of this section is devoted to proving sufficiency of the above necessary conditions. Since $k$ divides $n m^{2}$, we can write $k=n^{\prime} s^{2} t$, where $n^{\prime}, s, t \geqslant 1, n^{\prime}$ divides $n$, st divides $m$ and $t$ is square-free. Note that since $k$ is odd, we necessarily have that $n^{\prime}, s$ and $t$ are odd.

We break our proof into the following cases:

Case 1: $s=1$,

Case 2: $s>1$.

\subsection{The case $s=1$}

We split the case $s=1$ into the following subcases:

Case 1A: $t=1$,

Case 1B: $\quad t>1$.

Case 1A: $t=1$. Since $k=n^{\prime} s^{2} t$, we have that $k=n^{\prime}$. But as $k \geqslant n$ and $n^{\prime}$ divides $n$, we must have that $k=n$. A decomposition of $C_{n} * \bar{K}_{m}$ into copies of $C_{n}$ follows immediately from Theorem 4 (i).

Case 1B: $t>1$. If $n^{\prime}=n$, we can proceed as in Case 1A. Thus we may assume that $n / n^{\prime}>1$. Since $n \leqslant k=n^{\prime} t, n / n^{\prime} \leqslant t$. So from Lemma 4 , there exists a weak $\left(n / n^{\prime}, t, \mathbb{Z}_{t}\right)^{*}$-path. We next apply Lemma 2 to obtain a decomposition of $C_{n} * \bar{K}_{t}$ into cycles of length $k=n^{\prime} t$. Next, apply Theorem 4 (i) to obtain a decomposition of $C_{k} * \bar{K}_{m / t}$ into cycles of length $k$. Combining these results, we obtain a decomposition of $C_{n} * \bar{K}_{m}$ into cycles of length $k$, as required.

\subsection{The case $s>1$}

We split the case $s>1$ into the following subcases, depending on the value of $n / n^{\prime}$ :

Case 2A: $n / n^{\prime}<s$,

Case 2B: $s \leqslant n / n^{\prime} \leqslant s^{2}$ and

Case 2C: $s^{2}<n / n^{\prime}$.

We first deal with Case 2A. This is the most straight-forward of subcases in this section, as we are applying theory already developed in other papers.

Case 2A: $n / n^{\prime}<s$. Our goal is to decompose $C_{n} * \bar{K}_{s}$ into closed trails, each of length $n^{\prime} s^{2}$, so that the maximum degree of each vertex in each closed trail is at most $2\left\lceil n^{\prime} s / n\right\rceil$. Since $n m \geqslant k=n^{\prime} s^{2} t$, we have that $m /(t s) \geqslant n^{\prime} s / n$. Theorem 6 then tells us $C T *$ $\bar{K}_{m /(t s)}$ decomposes into cycles of length $n^{\prime} s^{2}$, for each of our closed trails $C T$. It follows immediately that $C_{n} * \bar{K}_{m / t}$ decomposes into cycles of length $n^{\prime} s^{2}$. We then apply Theorem 4 (ii) to obtain the required decomposition of $C_{n} * \bar{K}_{m}$ into cycles of length $k=n^{\prime} s^{2} t$. 
Let us now work on our initial goal. Write $n^{\prime} s=a n+2 b$, where $a \geqslant 1, a$ is odd and $0 \leqslant b<n$. We start with a decomposition of $C_{n} * \bar{K}_{s}$ into $s$ Hamilton cycles of length $n s$, using Theorem 5 as $s$ is odd. Consider the edges of two of these Hamilton cycles between a pair of adjacent partite sets. From Theorem 5, these edges form a 2-factor on $2 s$ vertices.

We take $2 b / n^{\prime}$ of the Hamilton cycles in our decomposition, pairing them to obtain $b / n^{\prime} 2$-factors between each adjacent pair of partite sets. There are $s-\left(2 b / n^{\prime}\right)=a n / n^{\prime}$ Hamilton cycles remaining.

We have a total of $n / n^{\prime}$ closed trails to create, each of length $n^{\prime} s^{2}$; we shall label them $C T_{1}, C T_{2}, \ldots C T_{n / n^{\prime}}$. We form $C T_{i}$ by taking $a$ of the Hamilton cycles and $b$ of the 2 -factors, making a total of ans $+2 b s=n^{\prime} s^{2}$ edges. However we choose the 2-factors in such a way so the maximum degree of a vertex in the closed trail is as small as possible. This is done by selecting 2 -factors between $V_{2 j+2 b i}$ and $V_{2 j+2 b i+1}$, for each $j, 0 \leqslant j \leqslant b-1$. The subscripts are as usual evaluated modulo $n$. Note the maximum degree of each closed trail is $2 a+2$ if $2 b<n$, or $2 a+4$ if $2 b>n$. In either case the maximum degree is at most $2\left\lceil n^{\prime} s / n\right\rceil$, as required.

Case 2B: $s \leqslant n / n^{\prime} \leqslant s^{2}$. The first step is to obtain a decomposition of $C_{n} * \bar{K}_{s}$ into cycles of length $n^{\prime} s^{2}$. If $n / n^{\prime}=s$, then such a decomposition follows from Theorem 4 (ii). If $s<n / n^{\prime} \leqslant s^{2}$, we apply Lemma 10 with $c=n / n^{\prime}$ to obtain a strong $\left(n / n^{\prime}, s, \mathbb{Z}_{s}\right)^{*}$-path $P$. We then apply Lemma 3 to obtain a decomposition of $C_{n} * \bar{K}_{s}$ into cycles of length $n^{\prime} s^{2}$.

Since $s t$ divides $m$, we can obtain a decomposition of $C_{n} * \bar{K}_{m}$ into cycles of length $k$, as required, by applications of Theorem 4 similar to previous cases. This completes Case 2B.

Before we commence the final Case $2 \mathrm{C}$, we introduce an alternative way of labelling vertices. Let $s$ and $t$ be positive integers. We label the vertices of $L_{\ell} * \bar{K}_{s t}$ with the elements of

$$
\left\{v_{(i, j)} \mid v \in \mathbb{Z}_{\ell+1}, i \in \mathbb{Z}_{s}, j \in \mathbb{Z}_{t}\right\},
$$

with an edge joining $u_{(i, j)}$ and $v_{\left(i^{\prime}, j^{\prime}\right)}$ if and only if $u$ and $v$ differ by 1 . We label the vertices of $C_{\ell} * \bar{K}_{s t}$ similarly.

As in the previous section, vertex labels in $C_{\ell} * \bar{K}_{s t}$ are calculated mod $\ell$ and $\oplus$ is defined as before. On the other hand, vertex subscripts are now calculated mod $(s, t)$. We (re-)define $G^{i}$ to be the graph created from $G$ by adding $i(\bmod t)$ to the second subscript only of each vertex of $G$. With this new labelling in mind we prove the following useful result.

Lemma 11. Suppose there exists a path $P$ within $L_{\ell} * \bar{K}_{\text {st }}$ with the following properties:

(P1) $P$ begins at vertex $0_{(0,0)}$ and ends at vertex $\ell_{(0,0)}$,

$(\mathrm{P} 2)$ if $P$ contains vertices of the form $0_{(i, j)}$ and $\ell_{(i, j)}$ then $(i, j)=(0,0)$, and

(P3) for each $i, j$ such that $0 \leqslant i, j \leqslant s-1$ and for each $d$ such that $0 \leqslant d \leqslant t-1$, there exists a unique edge of the form $\left\{v_{(i, \alpha)},(v+1)_{(j, \alpha+d)}\right\}$ within $P$ for some $v$ and $\alpha$. 
Then, for each $a \geqslant 1$, there exists a decomposition of $C_{a \ell} * \bar{K}_{\text {st }}$ into cycles of length as ${ }^{2} t$.

Proof. Concatenate the paths $P, P \oplus(l), P \oplus(2 l), \ldots, P \oplus((a-1) \ell)$ to obtain a cycle $C$ of length $a s^{2} t$ within $C_{a \ell} * \bar{K}_{s t}$. Then the set of graphs

$$
\left\{C^{x} \oplus y \mid 0 \leqslant x \leqslant t-1,0 \leqslant y \leqslant \ell-1\right\}
$$

constitute a decomposition of $C_{a \ell} * \bar{K}_{s t}$ into $a s^{2} t$-cycles.

We are now ready to proceed with the proof of the final Case $2 \mathrm{C}$.

Case 2C: $s^{2}<n / n^{\prime}$. Let $n / n^{\prime}=y+\sum_{i=1}^{s^{2}-1} x_{i}$, where $1<y \leqslant t$ and $x_{i} \in\{1, t\}$ for each $i=1,2, \ldots, s^{2}-1$. Note, this is possible since $n / n^{\prime}>s^{2}$ and as $k \geqslant n, s^{2} t \geqslant n / n^{\prime}$. Also, since $n / n^{\prime}, t$ and $s$ are odd, we may deduce that $y$ is also odd.

From Lemma 8 , there exists a strong $\left(s^{2}, s, \mathbb{Z}_{s}\right)^{*}$-path including the edge $\left[0_{0}, 1_{0}\right]$ which we denote by $Q$. Recall that we consider an edge of the form $\left\{j_{i},(j+1)_{i+d}\right\}$ to have difference $d$. Observe that within $Q$ there are precisely $s$ edges of each difference. Label the edges of $Q$ with $e_{1}, e_{2}, \ldots, e_{s^{2}}$, so that $e_{i}$ and $e_{i+1}$ share a common vertex for each $i$ such that $1 \leqslant i \leqslant s^{2}-1$ and $e_{1}=\left\{0_{0}, 1_{0}\right\}$.

We define a relation $\sim$ on the set of edges of $Q$ as follows. For edges $e, e^{\prime} \in Q$, we say that $e \sim e^{\prime}$ if $e=\left\{v_{\alpha},(v+1)_{\beta}\right\}$, and $e^{\prime}=\left\{w_{\beta},(w+1)_{\alpha}\right\}$, for some $\beta, \alpha, v$ and $w$. Thus every edge of difference 0 is related to itself, and excluding edges of difference 0 , the relation $\sim$ induces equivalence classes of size 2 which we shall call pairs.

We now create a $1-1$ mapping $f$ between the edges of $Q$ and the set $\left\{x_{i} \mid 1 \leqslant i \leqslant\right.$ $\left.s^{2}-1\right\} \cup\{y\}$. First, we let $f\left(e_{1}\right)=y$. Recall that each $x_{i}$ is equal to either $t$ or 1 . Let $T=\mid\left\{x_{i} \mid 1 \leqslant i \leqslant s^{2}-1\right.$ and $\left.x_{i}=t\right\} \mid$. Let $E^{\prime}$ be a set of $\min \left(\lfloor T / 2\rfloor,\left(s^{2}-s\right) / 2\right)$ pairs of edges, as defined in the previous paragraph. For each edge $e$ in a pair from $E^{\prime}$, we set $f(e)=t$. If either $T$ is odd or $\lfloor T / 2\rfloor>\left(s^{2}-s\right) / 2$ we repeatedly set $f(e)=t$ for unassigned edges $e$ of difference 0 , until precisely $T$ edges have been set to $t$. For all remaining edges $e$, we set $f(e)=1$. For each edge $e$, we refer to $f(e)$ as the weight of edge $e$.

We now use $Q$ and the weighting defined on its edges to create a path $P$ of length $s^{2} t$ in the graph $L_{n / n^{\prime}} * \bar{K}_{s t}$ which satisfies the conditions of Lemma 11. Since $s t$ divides $m$, we can then apply Theorem 4 (i) to obtain our required decomposition of $C_{n} * \bar{K}_{m}$ into $k$-cycles. So, it remains to show the existence of a path $P$ which satisfies (P1), (P2) and (P3).

Each edge $e_{i}$ of $Q$ with weight $w$ will be replaced by a path $P_{i}$ on $t$ edges spanning $w+1$ partite sets. We then concatenate paths $P_{1}, P_{2}, \ldots, P_{s^{2}}$ to form the required path $P$. Since $Q$ has $s^{2}$ edges and since $n / n^{\prime}=y+\sum_{i=1}^{s^{2}-1} x_{i}, P$ will have $s^{2} t$ edges and traverse $n / n^{\prime}+1$ partite sets, as required.

In general, whenever we replace an edge $e_{i}=\left\{v_{\alpha},(v+1)_{\beta}\right\}$ from $Q$ with a path $P_{i}$ of length $t, P_{i}$ begins at a vertex of the form $\left\{w_{(\alpha, 0)}\right\}$ and ends at a vertex of the form $\left\{w_{(\beta, 0)}^{\prime}\right\}$ for some $w$ and $w^{\prime}$. Moreover, for each path $P_{i}$ and for each $d \in \mathbb{Z}_{t}$, there will be a unique edge in $P_{i}$ of the form $\left\{v_{(\alpha, j)},(v+1)_{(\beta, j+d)}\right\}$ for some $j$. This, together with the structure of $Q$, will justify (P3). 
We start with edge $e_{1}$ from $Q$. From Lemma 4 , there exists a weak $\left(y, t, \mathbb{Z}_{t}\right)^{*}$-path $P^{\prime}$. We form path $P_{1}$ from $P^{\prime}$ by replacing each vertex of the form $v_{i}$ from $P^{\prime}$ with the vertex $v_{(0, i)}$. Note that from the conditions of Lemma 4 , there are no vertices of the form $v_{(0, i)}$ in $P_{1}$ for all $i$ such that $(t+1) / 2 \leqslant i \leqslant t-1$.

We next take care of all the paired edges which are weighted with $t$. Let $e_{i} \sim e_{j}$, where $i<j, f\left(e_{i}\right)=f\left(e_{j}\right)=t, e_{i}=\left\{v_{\alpha},(v+1)_{\beta}\right\}$ and $e_{j}=\left\{w_{\beta},(w+1)_{\alpha}\right\}$, for some $v$ and $w$.

Now, the idea is to replace $e_{i}$ with $2 t-1$ partite sets and $e_{j}$ with just one partite set. Note that in total $e_{i}$ and $e_{j}$ contribute $2 t$ partite sets so our above analysis on the number of partite sets used is not altered overall. We define $P_{i}$ to be the path:

$\left[z_{(\alpha, 0)},(z+1)_{(\beta, 1)},(z+2)_{(\alpha, 0)},(z+3)_{(\beta, 2)}, \ldots,(z+2 t-3)_{(\beta, t-1)},(z+2 t-2)_{(\alpha, 0)},(z+2 t-1)_{(\beta, 0)}\right]$,

where $z_{(\alpha, 0)}$ is the last vertex in the path $P_{i-1}$.

On the other hand, we define $P_{j}$ to be the path:

$$
\left[\left(z^{\prime}\right)_{(\beta, 0)},\left(z^{\prime}+1\right)_{(\alpha, 0)}\right]
$$

where $\left(z^{\prime}\right)_{(\beta, 0)}$ is the last vertex in the path $P_{j-1}$.

Next, consider an edge $e_{i}=\left\{v_{\alpha},(v+1)_{\alpha}\right\}$, of difference 0 weighted with $t$. Assuming that $z_{(\alpha, 0)}$ is the last vertex in the path $P_{i-1}$, we define $P_{i}$ to be the path:

$\left[z_{(\alpha, 0)},(z+1)_{(\alpha, 1)},(z+2)_{(\alpha, 0)},(z+3)_{(\alpha, 2)}, \ldots,(z+t-2)_{(\alpha,(t-1) / 2)},(z+t-1)_{(\alpha, 0)},(z+t)_{(\alpha, 0)}\right]$.

Finally, we must deal with edges weighted with 1 . We have to be slightly careful when two adjacent edges are weighted with 1 . We proceed as follows. We describe two different choices for the path $P_{i}$, where $e_{i}=\left\{v_{\alpha},(v+1)_{\beta}\right\}$ is of weight 1 . We label the choices for $P_{i}$ positive and negative.

For $P_{i}$ positive:

$$
\left[z_{(\alpha, 0)},(z+1)_{(\beta,(t-1) / 2)}, z_{(\alpha, 1)},(z+1)_{(\beta,(t-3) / 2)}, \ldots,(z+1)_{(\beta, 1)}, z_{(\alpha,(t-1) / 2)},(z+1)_{(\beta, 0)}\right],
$$

where $z$ is the final vertex used in path $P_{i-1}$. For $P_{i}$ negative:

$$
\left[z_{(\alpha, 0)},(z+1)_{(\beta,(t+1) / 2)}, z_{(\alpha, t-1)},(z+1)_{(\beta,(t+3) / 2)}, \ldots,(z+1)_{(\beta, t-1)}, z_{(\alpha,(t+1) / 2)},(z+1)_{(\beta, 0)}\right],
$$

where $z$ is defined as above.

To ensure that $P$ is indeed a path, we must take care that consecutive edges from $Q$ of weight 1 are not replaced with both positive or both negative paths. Moreover, to validate (P2) we need to make sure that if $f\left(e_{s^{2}}\right)=1$ then $P_{s^{2}}$ is negative. (Since, from above, there are no vertices in $P_{1}$ of the form $v_{(0, i)}$, for all $i$ such that $(t+1) / 2 \leqslant i \leqslant t-1$.) It is clearly possible to satisfy both of these conditions.

\section{References}

[1] B. R. Alspach and H. J. Gavlas, Cycle decompositions of $K_{n}$ and $K_{n}-I$, J. Combin. Theory Ser. B 81 (2001), 77-99. 
[2] E. J. Billington, N. J. Cavenagh and B. R. Smith, Path and cycle decompositions of complete equipartite graphs: 3 and 5 parts, Discrete Math. 310 (2010), 241-254.

[3] E. J. Billington, N. J. Cavenagh and B. R. Smith, Path and cycle decompositions of complete equipartite graphs: 4 parts, Discrete Math. 309 (2009), 3061-3073.

[4] D. Bryant and D. Horsley, An asymptotic solution to the cycle decomposition problem for complete graphs, J. Combin. Theory Series A, (to appear: doi:10.1016/j.jcta.2010.03.015).

[5] D. Bryant and D. Horsley, Decompositions of complete graphs into long cycles, Bull. Lond. Math. Soc. 41 (2009), 927-934.

[6] N. J. Cavenagh, Decompositions of complete tripartite graphs into $k$-cycles, Australas. J. Combinatorics 18 (1998), 193-200.

[7] N. J. Cavenagh and E. J. Billington, Decompositions of complete multipartite graphs into cycles of even length, Graphs and Combinatorics 16 (2000), 49-65.

[8] J. Liu, A generalization of the Oberwolfach problem and $C_{t}$-factorizations of complete equipartite graphs, J. Combin. Designs 8 (2000), 42-49.

[9] J. Liu, The equipartite Oberwolfach problem with uniform tables, J. Combin. Theory Series A, 101 (2003), 20-34.

[10] R.S. Manikandan and P. Paulraja, $C_{p}$-decompositions of some regular graphs, Discrete Math. 306 (2006), 429-451.

[11] M. Šajna, Cycle decompositions III: Complete graphs and fixed length cycles, J. Combin. Designs 10 (2002), 27-78.

[12] B. R. Smith, Complete equipartite 3p-cycle systems, Australas. J. Combin. 45 (2009), $125-138$.

[13] B. R. Smith, Decomposing complete equipartite graphs into cycles of length $2 p, J$. Combin. Designs 16 (2008), 244-252.

[14] D. Sotteau, Decomposition of $K_{m, n}\left(K_{m, n}^{*}\right)$ into cycles (circuits) of length $2 k, J$. Combin. Theory Ser. B 30 (1981), 75-81. 